\title{
Application of Remote Sensing Technology to the Ecology Quality Evaluation of Opencast Mine Area
}

\author{
Guojin He and Yan Peng
}

\begin{abstract}
In this paper, the rare earth mining area of southern Ganzhou in Jiangxi Province, China is taken as a study area. And the ecology quality evaluation mode based on remote sensing technology was built up to assess the ecology equality status of the opencast mine area. In order to determine the influence sphere on the surrounding ecology quality, the buffer zone method was introduced. The result shows that the rare earth mine exploitation has negative effects on vegetation coverage, biomass, water and soil loss and landscape. The ecology quality status became worse from 2000 to 2005 , while better from 2005 to 2010 by comprehensively analyzing the ecology quality status in the study area. And the influence on the ecology quality status caused by rare earth mine exploitation is very great in zone $1500 \mathrm{~m}$, while very little out of zone $2500 \mathrm{~m}$. It suggests that the influence sphere on the surrounding ecology quality of rare earth mine exploitation is about $2500 \mathrm{~m}$.
\end{abstract}

Index Terms-Opencast mine area, remote sensing, ecology quality evaluation, buffer zone, influence sphere.

\section{INTRODUCTION}

Mineral Resource plays an important role in national economy and social development of our country. On one hand, the exploitation of mineral resource contributes to the development of national economy. On the other hand, it caused various ecological environment problems, such as water and soil pollution, soil erosion and so on. These problems have restricted regional sustainable development of social economy in a certain degree. It is of great significance to evaluate the ecological quality of opencast mine area. But some data are very difficult to collect with traditional ecology quality assessment technique. In recent years, with the rapidly development of Remote Sensing technology, multi-resolution, multi-spectral, multi-platform and multi-temporal data further enriches, remote sensing techniques have become one of the most important means in eco-environment researches. In 2006, the Environmental protection industry of the People's Republic of China released a technical criterion for eco-environmental status evaluation which provides a certain guidance and reference for monitoring ecological environmental status using remote sensing and GIS [1]. Some assessed the ecological and environmental quality for a large area like a province or a city using the remote sensing technology [2]-[4]. And many researchers also applied remote sensing to monitoring

Manuscript received August 24, 2015; revised November 10, 2015. This work was supported by the Ecological Status and Ten Years Change of the Typical Area of Mineral Resources Exploitation Project under grant number STSN-10-03.

Guojin He and Yan Peng are all with the Institute of Remote Sensing and Digital Earth, Chinese Academy of Science, Beijing, China (e-mail: hegj@radi.ac.cn, pengyan@ radi.ac.cn). open-cast mine area. Anderson investigate the open-cast mine exploitation status with Landsat imageries and proposed that traditional method for monitoring the open-cast mine wasn't suitable for large-scale monitoring which takes lots of time and energy, and this is the reason why satellite remote sensing data is widely applied in the field [5]. Nuray Demirel investigates the use of SVM classification methods for identifying quantifying and analyzing spatial response of landscape due to surface mining activities in Goynuk open cast mine, Turkey, from year 2004 to 2008 [6]. Niu et al use SPOT, Landsat TM and aerial image for Jiangou mountain of Ningxia, Haibowang mining area of Neimenggu and Datong mining area of Shanxi respectively to determine the position and exploitation scale, and point out that using remote sensing and spatial technique can monitor the status of small coal mine well [7]. Li et al. investigated the geological disasters caused by coal mine exploitation, and analyzed the status, cause and distributed regularity of coal mine geological disasters status [8]. Peng choose rare earth mine of Dingnan as a study case, and use ALOS image to extract two different kinds of exploitation technique mining areas which are in-situ leaping method and non in-situ leaping method including pond leaching and heap leaching way respectively, and quantitatively analyzed the exploitation status, it pointed that the remote sensing image with $2.5 \mathrm{~m}$ resolution can identify clearly the ion-absorbed rare earth mine [9]. While there is less study on the ecology quality comprehensive assessment for open-cast mining area using remote sensing technology. And in the past research, many researchers focused on the open-cast mining area information abstraction or exploitation status monitoring, and rarely quantitatively analyzed the ecology quality and influence sphere on the surrounding. However, if the influence sphere of mine exploitation activities can be determined, it will provide more intuitive and precise governance goal for relative administration department and decision-makers, it has a great significance. Therefore, remote sensing technology was applied in this paper to evaluate the ecology quality for rare earth mine area considering the characteristics of the surrounding ecosystem destroyed by rare earth mine exploitation in southern Ganzhou. And the buffer zone method is introduced to determine the influence sphere of mine exploitation activities based on the ecology quality status evaluation.

\section{StUdy AREA AND DATA Processing}

\section{A. Study Area and Datasets}

Southern Ganzhou is located in the south of Ganzhou city, Jiangxi Province, which includes Dingnan county, Longnan county, Xinfeng county, Quannan county and Anyuan county (see Fig. 1). The study area is hilly, and the total area is about 
$9732.86 \mathrm{~km}^{2}$. The study area is one of the maize zone of Gannan navel oranges, and is rich in mineral resources especially in rare earth ore, and has become one of the first national rare earth ore planning counties. The rare earth mine of study area belongs to the south ion-absorbed elements type And the south ion-absorbed REM areas were scattered in study areas with small scale. In order to get a high accurate assessment result, images with good quality and high spatial resolution were chosen. Synthesizes the purpose, cost and the demand for information of study area, ALOS images, SPOT-5 and SPOT $1 / 2 / 4$ images were chosen to generate land use and land cover classification of 2010, 2005 and 2000 respectively, Landsat series images and ASTER DEM data were also collected to extract relative ecological quality evaluation index of 2000, 2005 and 2010 respectively. It involves 18 ALOS images which mainly acquired on November 2010, 10 SPOT-5 images mainly acquired on November, 2005, 6 images mainly acquired on November, 2001, 4 Landsat ETM+ images mainly acquired on October, 2001, 4 Landsat 5 images mainly acquired on Nov, 2005, and 4 Landsat 5 images mainly acquired on March, 2010.

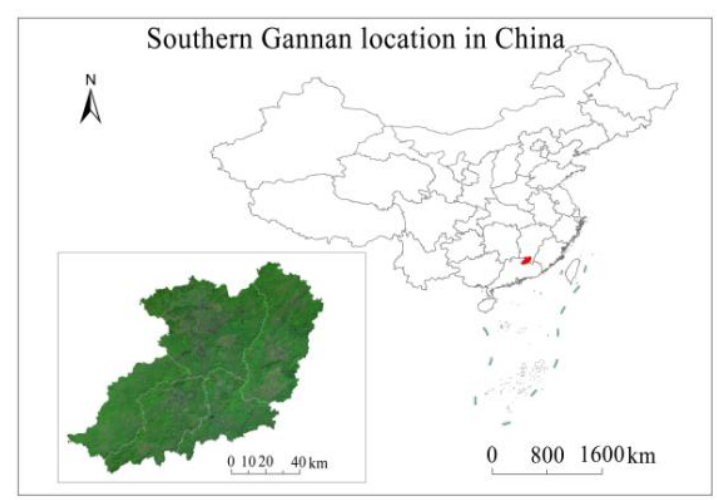

Fig. 1. Location map of southern Ganzhou.

\section{B. Data Processing}

Ortho-rectification, data fusion, image mosaic, image cut and image classification are should be carried out to extract the ecological quality index based on remote sensing. The ALOS data was orthorectified by Rational Function Model with RPC, and the SPOT series data was orthorectified by physical imaging model. The errors of the orthorectified images are within 1 pixel. The fused images were obtained using PANSHARP transform which is a popular fusion algorithm recently, and found the matching gray value between the fused bands mainly using the least squares method, and adjust the contribution values of individual band to keep its color best and to make it be close to natural colour, and estimated the gray value relationship among all bands using statistical method to remove the data gap problem [10], [11].

Image classification is a necessary step to assessment the ecology quality status using remote sensing technology. In this paper, the land use classification type of the study areas includes forest, bareland, water, farmland, building and rare earth ore mining area. For high spatial resolution image, the object-oriented classification way is more suitable than traditional classification method based on pixel. Therefore, an objects-oriented classification approach combining with object size, context features and texture information extracted by Gray Level Co-occurrence Matrix (GLCM) was applied in this study. The method can fully dig the spectral information, spatial characteristics and context information so that it can make the classification result fit better with the actual situation. For different periods, a backtracking method was introduced to obtain the change information and then generate classification of study area for other periods. The method can induced false changes efficiently, improve the accuracy and detect object changes for different years rapidly. Fig. 2 shows the classification result of 2000, 2005 and 2010 in the study area. In additional, field validation method combining with high spatial resolution image was applied to verify the classification accuracy. And the overall classification accuracy is about $90 \%$, Kappa value is over than 0.85 . The accuracy can meet the research's need.

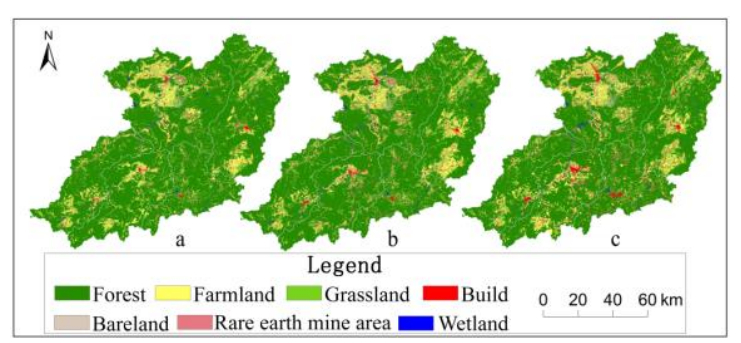

Fig. 2. Classification result of rare earth mining area in the southern Ganzhou, Jiangxi province, China. a, b, c represent results of 2000, 2005 and 2010 respectively.

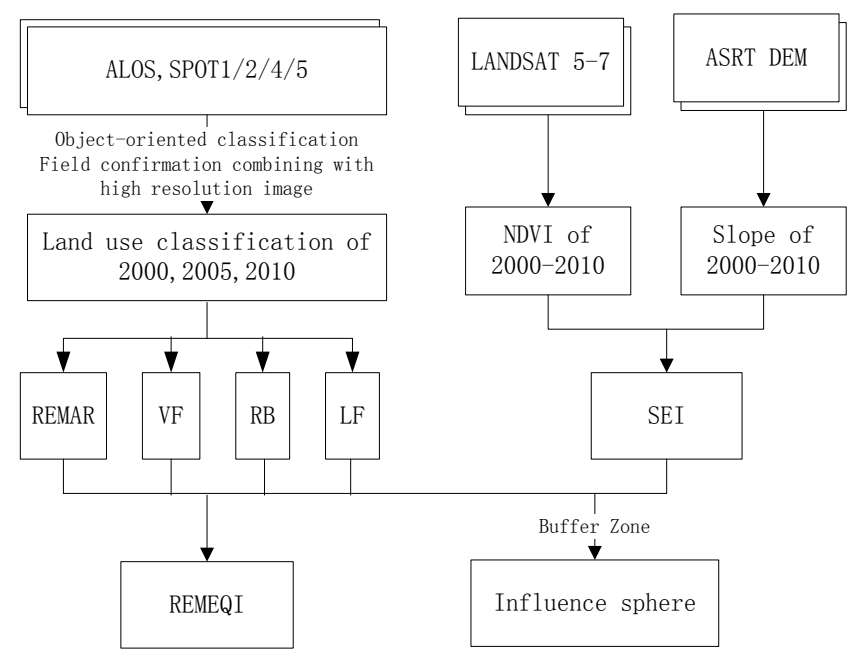

Fig. 3. The flow chart of ecology quality status evaluation

\section{REgional ECOLOGY QuALity Evaluation}

The rare earth mine exploitation activity made the landscape more broken, destroys the surface vegetation seriously and aggravates the land gradation due to its scattered distribution and small scale in the study area. In consideration of the characteristics of the surrounding ecosystem destroyed by rare earth mine exploitation, referring to the relative literatures about regional ecology quality assessment, and following the operable principle in indices selection processing, rare earth mine area rate (REMAR), vegetation fraction (VF), regional biomass (RB), soil erosion intensity index (SEI) and landscape fragmentation (LF) are chosen to reflect the ecology quality status in southern Ganzhou. Fig. 3 shows the flow of analysis 
the influence on the surrounding caused by rare earth mine exploitation in the study area.

\section{A. Rare Earth Mine Area Rate}

\section{1) Rare earth mine area rate}

Rare earth mining area rate can reflect the damage on ecosystem type caused by rare earth mine exploitation. The Rare earth mining area rate (REMAR) can be expressed as below.

$$
\mathrm{REMAR}=\frac{S_{r e m}}{S}
$$

Here, $S_{r e m}$ represents the rare earth mine exploitation area, $S$ is the area of the whole region.

\section{2) Vegetation fraction}

Vegetation Fraction is one of the indicators which can reflect the vegetation cover degree of a study area, and it is also the required parameters for calculating soil erosion intensity. And there are many ways to calculate vegetation fraction. Some estimated vegetation fraction using normalized different vegetation index (NDVI)[12], and some estimated it with the ecological system classification [1]. In this paper, the vegetation fraction was calculated using the classification mainly by reference to Technical Criterion for Eco-environmental Status Evaluation (on try) (HJ/T192-2006) [1]. And the expression is as equation 2 shows.

$$
V F=(0.38 \times f r+0.34 \times g+0.19 \times f l+0.07 \times b u+0.02 \times b l) / \mathrm{S}
$$

where $f r, g, f l, b u, b l$ represent the area of forest, grassland, farmland, building and bareland, and the rare earth mining area is regard as bareland in this calculating process due to the factor that the vegetation cover degree in rare earth mining area is similar to bareland. $V F$ is vegetation fraction.

\section{3) Regional ecosystem biomass}

In this study, the biomass per area index is applied to reflect the ecological system biomass in the study area, the expression is as equation 3 show.

$$
R B=\frac{\sum_{j=1}^{m} \sum_{i=1}^{n}\left(R B D_{i j} \times S_{p}\right)}{S}
$$

Here, $R B$ represents the ecosystem biomass in the study area. $\mathrm{j}$ is the ecosystem type which includes forest, grassland and wetland. $R B D_{i j}$ represents the relative biomass density of pixel $i$ in ecosystem type $\mathrm{j}$ which was provided by the special groups for Investigation and Evaluation of Ten Years Change of National Ecological Environment (2000-2010).

\section{4) Soil erosion intensity}

Soil erosion intensity and vegetation fraction are inversely proportional relationship under other constant conditions. Soil erosion intensity is calculated using vegetation fraction and slope factors in Standards for Classification and Gradation of Soil erosion [13]. In this paper, the soil erosion intensity is classified as slight erosion, moderate erosion and severe erosion according to the standards and considering the actual situation of the study area. Considering the ecological system types, the soil erosion intensity was calculated using fraction and slope factors for forest land, grass land, farm land, bare land and rare earth ore mining area, as Table I show. The soil erosion of other types including water area and building land are identified as inapparent soil erosion

\begin{tabular}{|c|c|c|c|c|c|c|}
\hline \multirow[t]{2}{*}{ VF } & \multicolumn{6}{|c|}{ Slope $\left(^{\circ}\right)$} \\
\hline & $<5$ & $5 \sim 8$ & $8 \sim 15$ & $15 \sim 25$ & $25 \sim 35$ & $>35$ \\
\hline$>75$ & Sli & Sli & Sli & Sli & Sli & Sli \\
\hline $60 \sim 75$ & Sli & Sli & Sli & Sli & Mod & Mod \\
\hline $40 \sim 60$ & Sli & Sli & Sli & Mod & Mod & Sev \\
\hline $30 \sim 45$ & Sli & Sli & Mod & Mod & $\mathrm{Sev}$ & $\mathrm{Sev}$ \\
\hline$<30$ & Sli & Mod & Mod & Sev & $\mathrm{Sev}$ & $\mathrm{Sev}$ \\
\hline
\end{tabular}
areas.

TABLE I: GRADATION TABLE OF SOIL EROSION INTENSITY

$\overline{\text { Sli, Mod and Sev present slight, moderate and severe erosion respectively. }}$

In order to quantitatively assess the soil erosion degree, the three gradations are given different weights respectively [1], as Table II shows.

TABLE II: THE WEIGHTS OF SOIL EROSION INTENSITY

\begin{tabular}{llll}
\hline \hline Type & Slight & Moderate & Severe \\
\hline Weights & 0.05 & 0.25 & 0.7 \\
\hline \hline
\end{tabular}

And the soil erosion intensity (SE) expression is as equation 4 shows:

$$
S E=\frac{0.05 S_{s l}+0.25 S_{m o}+0.7 S_{h i g}}{S}
$$

where, $S_{s l}, S_{m o}, S_{h i g}$ represent the slight, moderate and severe area respectively.

\section{5) Landscape fragmentation}

Landscape fragmentation is identified as the broken degree divided by the natural or human factors. The broken of landscape makes the plaque area become smaller, and will directly affect the biological diversity. At the same time, landscape fragmentation can also reflect the influence degree of human activities on landscape [14]. The index can be calculated by many ways [15-[17], and the total plaques numbers in unit area is used as the evaluation index of landscape fragmentation in this paper [17], as equation 5 shows.

$$
C=\frac{\sum n_{i}}{S}
$$

Here, $C$ represents the landscape fragmentation, $\sum n_{i}$ is the total plaques numbers in the whole region.

Table III lists each index value calculated by above equations respectively in 2000, 2005 and 2010. The rare earth mining area rate still increased from 2000 to 2010, which increased rapidly from 2000 to 2005, while slowly from 2005 to 2010. This is caused by reinforcing the reclamation after 2005. The vegetation fraction decreased 
from 2000 to 2010, which indicates that the vegetation coverage turned worse. The reason is that rare earth mine exploitation can destroy the surface vegetation seriously, even change the landscape, especially the pond leaching and heap leaching exploitation methods which is similar to "move mountain spot"[9]. The regional ecosystem biomass in the study area also reduced from 2000 to 2010, while the landscape fragmentation increased. It suggests that influence intensity of human activity on the ecosystem is aggrandized. And the rare earth mine exploitation activity is the strongest except the town expansion. The soil erosion intensity is aggrandized from 2000 to 2005, while weakened from 2005 to 2010. It indicates that the water and soil loss phenomena may be improved after 2005 due to reinforcing the governance for the abandon mining area in the mining area. In conclusion, rare earth mine exploitation has negative effects on vegetation coverage, biomass, water and soil loss and landscape.

TABLE III: INDEX VALUE OF EACH EVALUATION INDEX

\begin{tabular}{llllll}
\hline \hline Year & REMAR & VF & RB & SI & LF \\
\hline 2000 & 0.0037 & 0.3408 & 0.3456 & 0.1459 & 947.00 \\
2005 & 0.0050 & 0.3364 & 0.2846 & 0.1990 & 1200.61 \\
2010 & 0.0051 & 0.3344 & 0.2962 & 0.1449 & 1215.79 \\
\hline \hline
\end{tabular}

\section{B. Regional Ecology Quality}

The ecological quality assessment model in rare earth mining area is built by weighting the above indexes, and can be expressed as equation 6 .

$$
\text { REMEQI }=\sum_{i}^{n} \omega_{i}\left(\frac{100}{\left(A_{i}\right)_{\max }} A_{i}\right)
$$

where, REMEQI represents the rare earth mine ecological quality index. $\omega_{i}$ is the weight of index $i$ including rare earth mining area rate, vegetation fraction, soil erosion intensity, biomass per area and landscape fragmentation, and it was obtained as Table IV showing using analytic hierarchy process (AHP) software according to the relative important degree of individual assessment index. $A_{i}$ is a value of index

$i,\left(A_{i}\right)_{\max }$ is the maximum value among these indexes, and $\frac{100}{\left(A_{i}\right)_{\max }}$ signifies the normalization processing for these indexes, and the normalization value should range from 0 to 100.

TABLE IV: WEIGHTS OF EACH ASSESSMENT INDEX

\begin{tabular}{llllll}
\hline \hline Index & REMAR & VF & RB & SI & LF \\
\hline Weights & 0.2 & 0.21 & 0.28 & 0.12 & 0.19 \\
\hline \hline
\end{tabular}

TABLE V: ECOSYSTEM QUALITY INDEX VALUE IN 2000, 2005 AND 2010

\begin{tabular}{llll}
\hline \hline Index & 2000 & 2005 & 2010 \\
\hline REMEQI & 61.8928 & 44.4168 & 47.8596 \\
\hline \hline
\end{tabular}

Table V lists the ecosystem quality index value in 2000 , 2005 and 2010 estimated by the above model. The ecosystem quality is best in 2000, and worst in 2005, while turns better in 2010. The change trend fits well with the rare earth mine exploitation status from 2000 to 2010. And the analysis result shows that the governance for rare earth mining area has achieved a certain success in southern Ganzhou at least.

\section{INFLUENCE SPHERE}

Buffer zone method was introduced on basis of the regional ecosystem quality assessment to determine the sphere of influence on the surrounding ecology quality caused by rare earth mine exploitation. It is a way that different distance buffers are built up centred on the rare earth mining area boundary, and then the ecological quality of each buffer zone is evaluated respectively. When the ecological quality index value begins to flat beyond a certain zone, the zone is regarded as the sphere of influence on the surrounding ecological quality caused by rare earth mine exploitation. In this paper, the buffer zone interval shouldn't be too large because of the small mining scale and scattered distribution of rare earth mining area. Then zones with $500 \mathrm{~m}$, $1000 \mathrm{~m}, 1500 \mathrm{~m}, 2000 \mathrm{~m}, 2500 \mathrm{~m}, 3000 \mathrm{~m}$, and $4000 \mathrm{~m}$ are established respectively centred on the rare earth mining area boundary in 2010. Fig. 4 shows the ecology quality status value for each buffer zone. The ecology quality tends to be better from zone $500 \mathrm{~m}$ to $4000 \mathrm{~m}$. The ecology quality status varied obviously from zone $500 \mathrm{~m}$ to $1500 \mathrm{~m}$, slowly from zone $1500 \mathrm{~m}$ to $2500 \mathrm{~m}$, and very little out of zone $2500 \mathrm{~m}$. The analysis result shows that the influence on the ecology quality status caused by rare earth mine exploitation is very great in zone $1500 \mathrm{~m}$, and begins to weaken in zone $1500 \mathrm{~m}-2500 \mathrm{~m}$, while the influence is very little out of zone $2500 \mathrm{~m}$. So we can include that the influence sphere on the surrounding ecology quality status caused by rare earth mine exploitation is about $2500 \mathrm{~m}$.

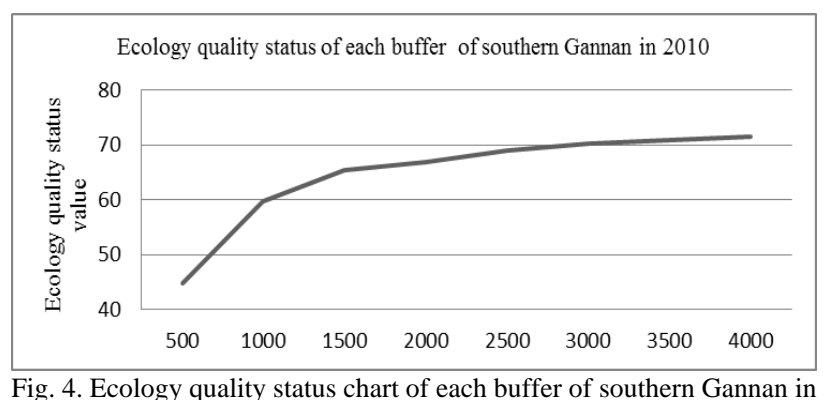

Fig. 4. Ecology quality status chart of each buffer of southern Gannan in 2010 .

\section{COnClusions}

Buffer zone method combining with the rare earth mine ecology quality evaluation method based on remote sensing technique can not only reflect the ecology quality status in the study area, but also determine the influence sphere on the surrounding ecology quality of rare earth mine exploitation. At the same time, all of these evaluated factors can be obtained through remote sensing data, which solved the problem that some data of a small region is difficult. The rare earth mine exploitation has negative effects on vegetation coverage, biomass, water and soil loss and landscape. The ecology quality status became worse from 2000 to 2005 , while better from 2005 to 2010 by comprehensively 
analyzing the ecology quality status in the study area. The analysis result shows that the governance for abandoned rare earth mining area has achieved a certain success in southern Ganzhou at least, and the success practices could provide guidance for the protection and governance of other rare earth mining area. At the same time, the influence on the ecology quality status caused by rare earth mine exploitation is very great in zone $1500 \mathrm{~m}$, while very little out of zone $2500 \mathrm{~m}$. It suggests that the influence sphere on the surrounding ecology quality of rare earth mine exploitation is about $2500 \mathrm{~m}$. This research will provide not only a support and decision-making for the relevant department to set up plans for mineral resource exploitation, but also a method and technical reference for the ecology quality assessment of other mineral resources mining area.

\section{REFERENCES}

[1] Technical Criterion for Eco-environmental Status Evaluation (On try), Environmental protection industry Standard HJ/T 192-2006.

[2] Y. Wang, H. L. Gong, and X. J. Li, "The Monitoring and analysis of ecological environment quality of Beijing based on GIS," Remote Sensing for Land and Resources, no. 1, pp. 91-97, 2008.

[3] Z. L. Jiang, J. M. Sha, and W. N. Yang, "Multiple factors-based on remote sensing evaluation of ecological environment in Fuzhou," Remote Sensing for Land and Resources, vol. 61, no. 3, pp. 46-48, 2004.

[4] S. X. Wang, Y. Zhou, Y. Yao, and X. D. Han, "Ecological environment condition evaluation mode of county region based on remote sensing techniques," China Environmental Science, vol. 32, no. 1, pp. 181-186, 2012.

[5] A. T. Anderson, D. Schultz, N. Buchman, and H. M. Nock, "Landsat imagery for surface mine inventory," Photogrammetric Engineering and Remote Sensing, no. 43, pp. 1027-1063, 1997.

[6] D. Nuray, M. K. Emil, and D. Sebnem, "Landsat imagery for surface mine inventory," Photogrammetric Engineering and Remote Sensing, no.43, pp. 1027-1063, 2011.

[7] B. R. Niu and S. X. Sun, "The application of SPOT satellite in coal geological survey," Coal Geology of China, vol. 11, no. 2, pp. 19-22, 1999.

[8] C. Z. Li, H. F. Nie, J. Wan, and H. Xiao, "A remote sensing study of characteristics of geological disasters in a mine," Remote Sensing for Land \& Resources, no. 1, pp. 45-49, 2005.

[9] Y. Peng, G. J. He, and H. Cao, "Extraction of rare earth mining area using objectes-oriented classification approach based on texture characteristics," Science Technology and Engineering, vol. 13, no. 9, pp. 5590-5596, 2013.

[10] H. Li, B. S. Manjunath, and S. K. Mitra, "Multesensor image fusion using the wavelet transform," Graphical Models and Image Processing, vol. 57 , no. 3, pp. 235-245, 1995.

[11] Z. Zhong and R. S. A. Blum, "Categorization of multiscale-decomposition-based image fusion schemes with a performance study for a digital camera application," in Proc. Institute of Electrical and Electronics Engineers, 1999, vol. 87, no. 8, pp. 1305-1326.

[12] M. M. Li, B. F. Wu, and C. Z. Yan, "Estimation of vegetation fraction in the upper basin of miyun reservior by remote rensing," Resources Science, vol. 26, no. 4, pp. 153-159, 2004.

[13] Standards for Classification and Gradation of Soil Erosion, Water Conservancy industry Standard of the People's Republic of China SL 190-2007.

[14] B. Q. Jia, L. J. Yang, H. Yang, L. Sun, and J. Q. Yang, "A study on the landscape pattern change of oasis in and land," Acta Ecological Sinica, vol. 21, no. 1, pp. 34-40, 2001.

[15] D. M. Song, D. N. Xiao, and Z. C. Zhang, "Landscape changes of Minqin oasis in Gansu Province and its driving force," Chinese Journal of Applied Ecology, vol. 14, no. 4, pp. 535-539, 2003.

[16] J. S. Deng, K. Wang, J. F. Xun, Z. Q. Shen, and Y. R. Gao, "Impacts of farmland consolidation on farmland landscape," Chinese Journal of Applied Ecology, vol. 17, no. 1, pp. 41-44, 2003.

[17] L. D. Chen and B. J. Fu, "Analysis of impact of human activity on landscape structure in yellow river delta - A case study of Dongying region," Acta Ecological Sinica, vol.16, no.4, pp. 337-344, 1996.

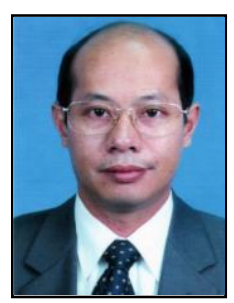

Guojin He was born in Fujian province, P. R. China, in 1968. He received the B.Sc. in geology from Fuzhou University in 1989 and the M.Sc. in remote sensing of geology from China University of Geosciences (Wuhan), in 1992. And he got the Ph.D. In geology from Institute of Geology, Chinese Academy of Sciences (CAS), in 1998. From 1992 to 2007, he worked at the Information Processing Department of China Remote Sensing Satellite Ground Station (RSGS), CAS. In 2001, He became the deputy director of Information Processing Department of RSGS, CAS. From 2004, he was a professor and director of Information Processing Department of RSGS, and also headed the research group of Remote Sensing Information Mining and Intelligent Processing. From 2008-2012, he was the professor and director of the Value-added Product Department, and the deputy director of the Spatial Data Center, Center for Earth Observation and Digital Earth, CAS. Since 2013, he has been the professor and director of the Satellite Data Based Value-added Product Department, and the deputy director of RSGS, Institute of Remote Sensing and Digital Earth, CAS. A large part of his earlier research dealt with information processing and applications of satellite remote sensing data. His current research interests are focusing on optical high resolution remote sensing image understanding as well as using information retrieved from satellite remote sensing images, in combination with other sources of data to support better understanding of the earth.

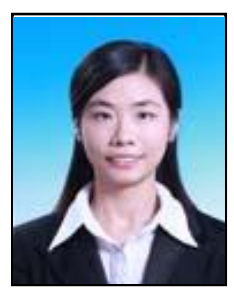

Yan Peng was born in Hunan, P.R. China, in 1988. She received the B.S. degree from University of South China, Hunan province, China in 2010, the M.S. degree from Institute of Remote Sensing and Digital Earth, Chinese Academy of Science, Beijin, China, in 2013. And she joined the Institute of Remote Sensing and Digital Earth, CAS, as a research assistant in 2013. Her current research interests are atmospheric correction and remote sensing applications. 